\title{
A novel in vitro periodontal pocket model to evaluate the effect of root surface instrumentation on biofilm-epithelial cell interactions
}

\author{
Kiri N. Lang ${ }^{1} \cdot$ Anton Sculean $^{1} \cdot$ Sigrun Eick $^{1} \cdot$ Alexandra Stähli $^{1}$
}

Received: 13 September 2021 / Accepted: 2 January 2022 / Published online: 19 January 2022

(c) The Author(s) 2022

\begin{abstract}
Objective To develop a novel in vitro periodontal pocket model for evaluating the effect of two different root surface instrumentation modalities on biofilm-epithelial cell interactions.

Materials and methods An artificial periodontal pocket model was created using an impression material. Dentin discs were prepared and incubated for 3.5 days with a biofilm consisting of 12 bacterial strains. Then, the discs were inserted into the pocket model and instrumented for $10 \mathrm{~s}$ or 10 strokes either with ultrasonics (US) or hand instruments (HI). Subsequently, a glass slide coated with epithelial cells was placed in close vicinity to the discs. After incubation of the pocket model in a 5\% $\mathrm{CO}_{2}$ atmosphere for $6 \mathrm{~h}$, residual bacteria of the biofilm as well as bacteria adhering to or invaded into epithelial cells were determined using colony-forming unit (cfu) counts and real-time PCR. Further, as a parameter of the pro-inflammatory cell response, interleukin (IL)-8 expression was determined by ELISA.

Results Compared to untreated control, HI reduced the cfu counts by $0.63 \log 10$ (not significant) and US by $1.78 \log 10$ $(p=0.005)$ with a significant difference between the treatment modalities favoring US $(p=0.048)$. By trend, lower detection levels of Tannerella forsythia were detected in the US group compared to HI. Concerning the interaction with epithelial cells, half of the control and the HI samples showed epithelial cells with attaching or invading bacteria, while US displayed bacteria only in two out of eight samples. In addition, US resulted in significantly lower IL-8 secretion by epithelial cells compared to the untreated control. Between HI and controls, no statistically significant difference in IL-8 secretion was found. Conclusion This newly developed in vitro model revealed in terms of biofilm-epithelial cell interaction after root surface instrumentation that compared to hand curettes, ultrasonic instrumentation appeared to be more effective in removing bacterial biofilm and in decreasing the inflammatory response of epithelium to biofilm.

Clinical relevance Ultrasonic instrumentation might be more advantageous to reduce cellular inflammatory response than hand instruments.
\end{abstract}

Keywords Biofilm-epithelial cell interactions $\cdot$ Periodontal pocket model $\cdot$ Root surface instrumentation

Alexandra Stähli

alexandra.staehli@zmk.unibe.ch

Kiri N. Lang

kiri.lang@zmk.unibe.ch

Anton Sculean

anton.sculean@zmk.unibe.ch

Sigrun Eick

sigrun.eick@zmk.unibe.ch

1 Department of Periodontology, School of Dental Medicine, University of Bern, Freiburgstrasse 7, 3010 Bern,

Switzerland

\section{Introduction}

Periodontitis is an opportunistic infectious disease caused by oral bacteria and their interplay with the intricacy of the host's immune and inflammatory response. Its primary features include the inflammatory-driven destruction of periodontal tissues resulting in periodontal pocketing, bleeding on probing, clinical attachment, and bone loss. The elimination of the biofilm is still the major goal of periodontal therapy (Löe et al. 1965; Salvi et al. 2012) and includes mechanical instrumentation-either manually or with ultrasonic instruments - of the exposed root surfaces. An alternative approach would be to influence the inflammatory response itself which is predominantly, if not entirely, responsible 
for tissue destruction. In the majority of cases, however, mechanical instrumentation is sufficient to induce healing of the periodontal tissues.

There has been a long lasting discussion on whether power-driven instruments (sonic and ultrasonic scalers) are to be preferred over hand instruments [8]. The consensus is that there is no difference in terms of clinical results between the two treatment modalities $[14,35]$ but that-in order to achieve an optimum - the combination of hand and ultrasonic instrumentation may be preferred [11]. Regarding substance loss, the least loss was noted for ultrasonic, then sonic, and finally hand instruments [29]. Thereby, hand curettes showed a mean substance loss per stroke of $6.8 \mu \mathrm{m}$ when low forces were applied and $20.6 \mu \mathrm{m}$ for high forces [37]. Ultrasonic instrumentation yielded smoother surfaces than the curette [7]. Regarding fibroblast survival and proliferation, no differences were found between hand curettes or ultrasonic systems [19]. In vitro models to investigate cell-biofilm interactions and therapeutic modalities are important tools in understanding not only the pathogenesis of periodontal disease but also its therapy. New experimental models could shed light on differences between ultrasonic and hand instrumentation in terms of microbiological and immune-inflammatory parameters.

In a previous study of our group, a similar in vitro pocket model was used that demonstrated considerable differences in biofilm removal and its reformation, surface alterations, and attachment of periodontal ligament fibroblasts when applying different non-surgical treatment modalities [12]. Hand curettes demonstrated the least biofilm reduction, while the use of an ultrasonic scaler resulted in the highest biofilm reduction. Surfaces after ultrasonication, in return, attracted the highest counts of PDL fibroblasts. Another group simulated the host-pathogen interplay in an in vitro multispecies biofilm model with Streptococcus mitis, Fusobacterium nucleatum, Porphyromonas gingivalis, and Aggregatibacter actinomycetemcomitans and oral epithelial cells to evaluate the effect of naturally derived polyphenol resveratrol and chlorhexidine [25]. Both compounds produced a downregulation of IL-8. An even more complex in vitro model mimicking the periodontal pocket challenged gingival epithelial keratinocytes, gingival fibroblasts, and monocytic cells (Mono-Mac 6) in a 3D collagen sponge with an 11-species biofilm [2]. As it was demonstrated, the cell conglomerate suppressed selective biofilm species implying an overall antimicrobial effect of the cells. Of course, their secretion of pro-inflammatory cytokines (IL-1 $\beta$, IL-2, IL-8, $\mathrm{TNF}-\alpha$ ) was significantly increased in the presence of the biofilm [2]. Another in vitro immune cell-gingival tissuebiofilm model suggests that gingival epithelium modulates the cytokine expression of immune cells and vice versa [4]. When gingival epithelium was co-cultured with monocytes, IL-8 was increasingly expressed in monocytes, while it was downregulated in gingival epithelium [4]. Increased expression of the antimicrobial cytokine CCL20 and the inflammatory cytokines CXCL8 (IL-8) and IL-6 was observed in a multi-layered gingival epithelium grown on a collagen hydrogel when exposed to different biofilms [5]. Interestingly, the inflammatory response was stronger when the tissue model was exposed to a commensal oral biofilm than to a gingivitis-associated one [5].

IL-8 is a major chemoattractant cytokine and activator of neutrophils in both health and disease. Tissues of patients with chronic periodontitis show higher levels of IL-8 which is expressed by various cell types at sites of inflammation [26]. Oral bacteria induce the expression of IL- 8 in epithelial cells. Whether lipopolysaccharide (LPS), a key virulence factor of gram-negative bacteria, also triggers IL-8 expression in epithelial cells is unknown. Recent data suggest that gingival epithelial cells might increase their responsiveness to LPS during episodes of dysbiosis and inflammation [18].

These aforementioned models were established to investigate host-microbiome interactions. However, there is limited evidence of models integrating biofilm-cell interactions in combination with therapeutic tools. As ultrasonic instrumentation uses water irrigation and is associated with less cementum removal, we hypothesized that manual and ultrasonic instrumentation may differ in the induced inflammatory response. Therefore, we sought to establish an in vitro biofilm-epithelial cell pocket model and to investigate how two different instrumentation modalities, hand and ultrasonic instrumentation, influence the biofilm removal and the interaction with epithelial cells.

\section{Materials and methods}

\section{Ethical approval}

Dentin discs were prepared from extracted human molar teeth that had been extracted for periodontal reasons during regular treatment from patients having given written informed consent. As teeth were anonymously collected, no ethical approval was required according to the guidelines set by the Ethics Committee of the University of Bern.

\section{Preparation of specimens and pocket model}

Out of the extracted teeth, dentin discs with the size of $5 \times 5 \times 1 \mathrm{~mm}$ were made by using a diamond burr and grinding turntables. Next, they were adhesively attached to plastic specimen holders with a dentin adhesive system (Syntac, Ivoclar Vivadent, Schaan, Lichtenstein). In order to expose the dentin discs to gingival epithelial cells, a pocket model was created where the specimen holder with the dentin disc (and biofilm) and another one with a glass slide 
(with epithelial cells) could be put in close contact to each other with a distance of $4 \mathrm{~mm}$. The pocket model was fabricated with a silicon dental impression material (Optosil, Kulzer GmbH, Hanau, Germany). The two specimen holders together with a spacer of 4-mm thickness were placed into the silicon mass and kept in situ during the hardening process. With this procedure, pockets of the same size were created. Pocket models were completely dipped in distilled water and autoclaved (Fig. 1).

\section{Biofilm formation}

The dentin discs on the plastic specimens were colonized with a biofilm consisting of 12 bacterial strains (Streptococcus gordonii ATCC 10,558, Actinomyces naeslundii ATCC 12,104, Fusobacterium nucleatum ATCC 25,586,
Campylobacter rectus ATCC 33,238, Eubacterium nodatum ATCC 33,099, Eikenella corrodens ATCC 23,834, Parvimonas micra ATCC 33,270, Filifactor alocis ATCC 33,099, Prevotella intermedia ATCC 25,611, Porphyromonas gingivalis ATCC 33,277, Tannerella forsythia ATCC 43,037, Treponema denticola ATCC 35,405). Bacterial strains (except for T. denticola) were cultivated on Schaedler Agar plates (Oxoid Basingstoke, UK) with 5\% sheep blood in an anaerobic atmosphere or with $5 \% \mathrm{CO}_{2} . T$. denticola was maintained in mycoplasma broth (BD, Franklin Lakes, NJ) supplemented with niacinamide, spermine tetrahydrochloride, and cocarboxylase in anaerobic conditions.

Dentin specimens were covered with $1.5 \%$ bovine serum albumin for $15 \mathrm{~min}$, before they were placed into tubes with nutrient broth (Wilkins-Chalgren broth with nicotinamide adenine dinucleotide and $\mathrm{N}$-acetyl muramic
Fig. 1 Illustration of the pocket model used for the experiments

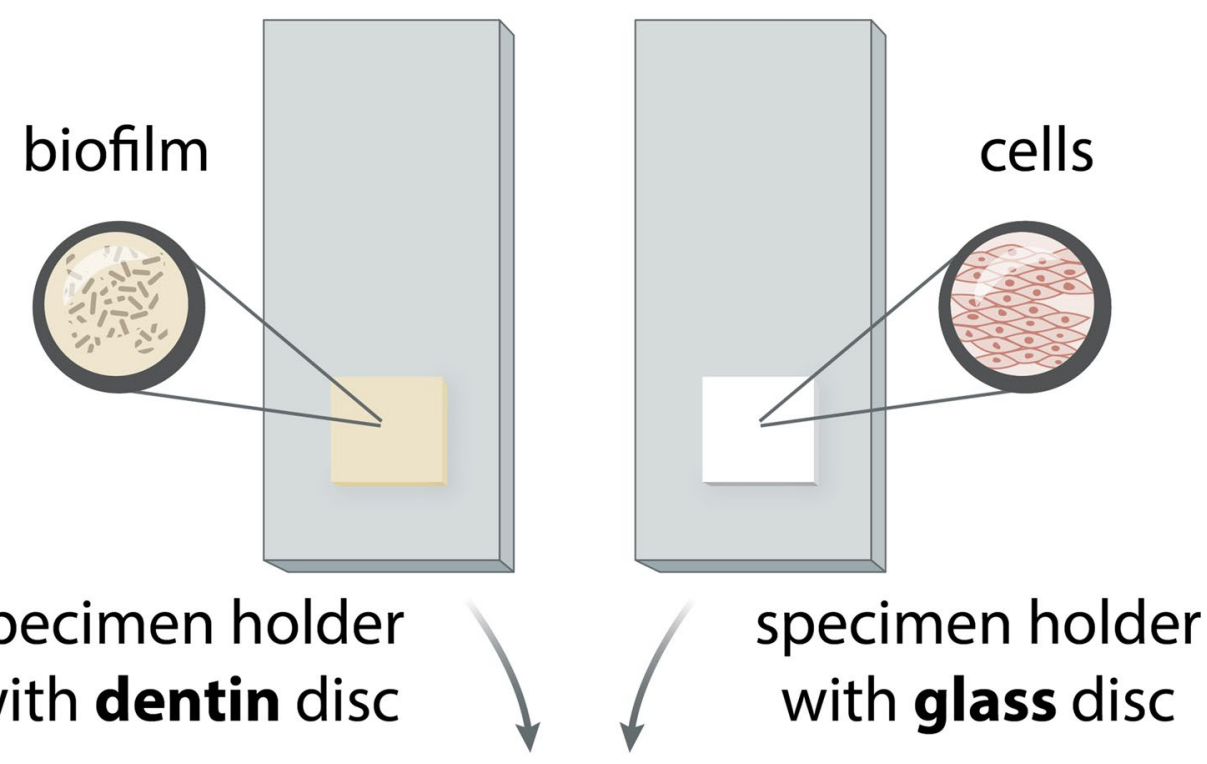

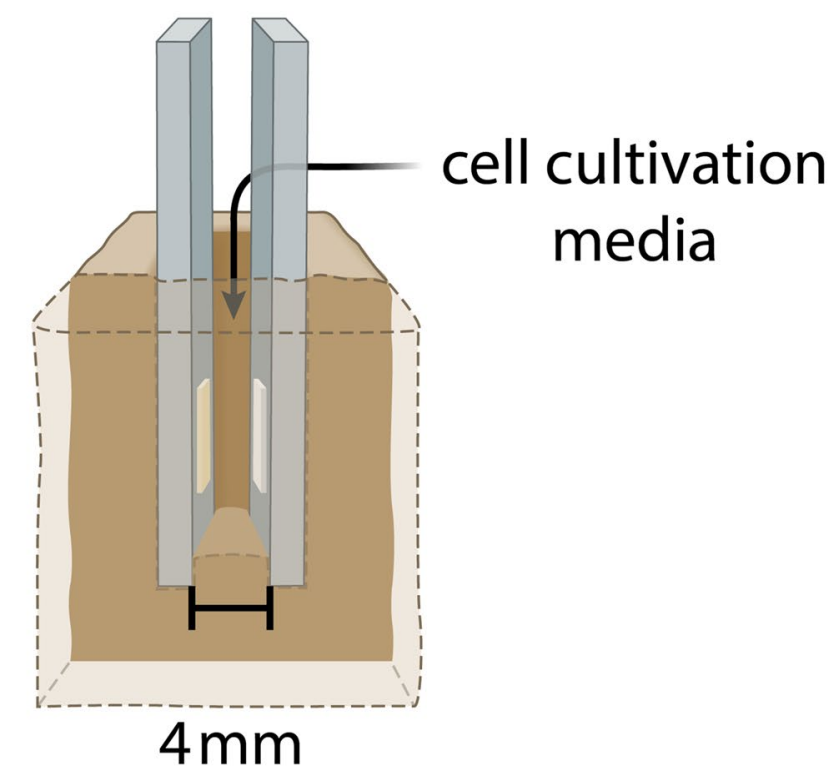


acid) and bacteria. The tubes have been incubated in anaerobic conditions at $37^{\circ} \mathrm{C}$ for 3.5 days. After $48 \mathrm{~h}, P$. gingivalis ATCC 33,277, T. forsythia ATCC 43,037, and T. denticola ATCC 35,405 were added again to guarantee a colonization of these bacteria in biofilm.

\section{Oral epithelial cells}

Thin glass slides of a size of $5 \mathrm{~mm} \times 5 \mathrm{~mm}$ were cut and fixed on a microscope slide. The glass slides were coated overnight with $0.01 \%$ poly-L-lysine solution (Sigma-Aldrich, Buchs, Switzerland). Then, telomeraseinactivated gingival keratinocyte (TIGK) cells (ATCCCRL-3397) in cell cultivation media (Keratinocyte Growth Medium, KGM-Gold, Lonza, Basel, Switzerland) were added. Before instrumentation, cells were checked that they had grown to a confluent monolayer.

\section{Instrumentation}

After 3.5 days of biofilm formation, specimens with the dentin discs and the biofilm were transferred from the tube to the silicon form of the periodontal pocket model. Then, two different treatment modalities were applied. Dentin specimens were scaled either with 10 strokes at average working pressure using $11 \mathrm{GC} 12$ Gracey hand curettes (Gracey curettes, Deppeler SA, Rolle, Switzerland) or an ultrasonic device (W\&H piezo scaler with W\&H tips $2 \mathrm{U}$ ) with water and power setting according to the manufacturer's instruction for $10 \mathrm{~s}$. All treatments were performed by an experienced periodontist (AS). A negative control with no applied treatment completed the groups.

\section{Exposure of treated biofilm to epithelial cells}

Biofilm-inoculated and then within the pocket modeltreated dentin specimens were shortly taken out of the silicon model, dipped into PBS, and transferred back to the mold. Then, the specimen holder with the TIGK cells was placed in the pocket model with the cells facing the treated dentin specimens at close distance of $4 \mathrm{~mm}$. Cell cultivation medium was added until both slides (with biofilm or TIGK cells) were covered. Thereafter, the periodontal pocket model with the treated biofilm and the TIGK cells has been incubated at $37{ }^{\circ} \mathrm{C}, 5 \% \mathrm{CO}_{2}$, and $95 \%$ humidity for $6 \mathrm{~h}$. Thereafter, supernatant was removed and kept frozen at $-80{ }^{\circ} \mathrm{C}$ until later assayed for IL-8 concentration. The specimens with the biofilm remains and with the epithelial cells were also taken out of the periodontal pocket model and processed further.

\section{Analysis of biofilms on dentin specimen}

Specimens with the dentin discs and the remained biofilm were shortly dipped into PBS. Thereafter, the biofilm was removed from the surface by intensive swabbing with a cotton swab which was given in a tube with PBS. After intensive mixing by pipetting and vortex, aliquots were spread on agar plates. Here, colony-forming units (cfu) were enumerated after an anaerobic incubation for 7 days. From another aliquot, DNA was extracted which was proceeded for quantification of $P$. gingivalis, $T$. forsythia, T. denticola, $P$. intermedia, F. nucleatum, and $C$. rectus counts by using real-time PCR as described before [9].

\section{Analysis of bacteria attached and invasive to epithelial cells}

Specimens with epithelial cells were shortly dipped into PBS and thereafter placed into ice-cold water for $15 \mathrm{~min}$. Then, after intensive mixing, aliquots were proceeded as before. This method quantifies the bacteria attaching to epithelial cells or being already invasive to the cells.

\section{Analysis of released interleukin-8}

The cell culture medium exposed to the treated biofilm and epithelial cells was analyzed for its IL- 8 concentration by using commercially available enzyme-linked immunosorbent assay (ELISA) kits (R\&D Systems, Minnesota, MN, USA) according to the manufacturer's instruction.

\section{Statistical analysis}

The final experiments were run in four series with each two specimen pairs (dentin, epithelium) per group. Statistical analysis was made by using SPSS 26.0 (IBM Corporation, New York, NY, USA). Groups were compared with the Kruskal-Wallis test followed by the Mann-Whitney test with Bonferroni correction. Significance was set at $p<0.05$.

\section{Results}

\section{Biofilm removal}

The cfu in biofilms $6 \mathrm{~h}$ after instrumentations differed statistically significantly $(p=0.004)$. The curettes reduced the counts by $0.63 \log 10$ (not significant) and US by $1.78 \log 10$ $(p=0.005)$ compared to the untreated control. In addition, there was a difference between the two treatment modalities in favor of US ( $p=0.048$; Fig. 2A).

Concerning the detection of single bacterial species by PCR $6 \mathrm{~h}$ after treatment, it was revealed that $P$. gingivalis was detected in all control biofilm samples and nearly in 
Fig. 2 Median, quartiles, and range of total colony-forming units (cfu; A) and counts of selected bacterial species (determined by real-time PCR; $\mathbf{B}$ ) of the remained biofilm on dentin discs $6 \mathrm{~h}$ after applying hand instrumentation (curette) and an ultrasonic scaler to biofilms on dentin discs

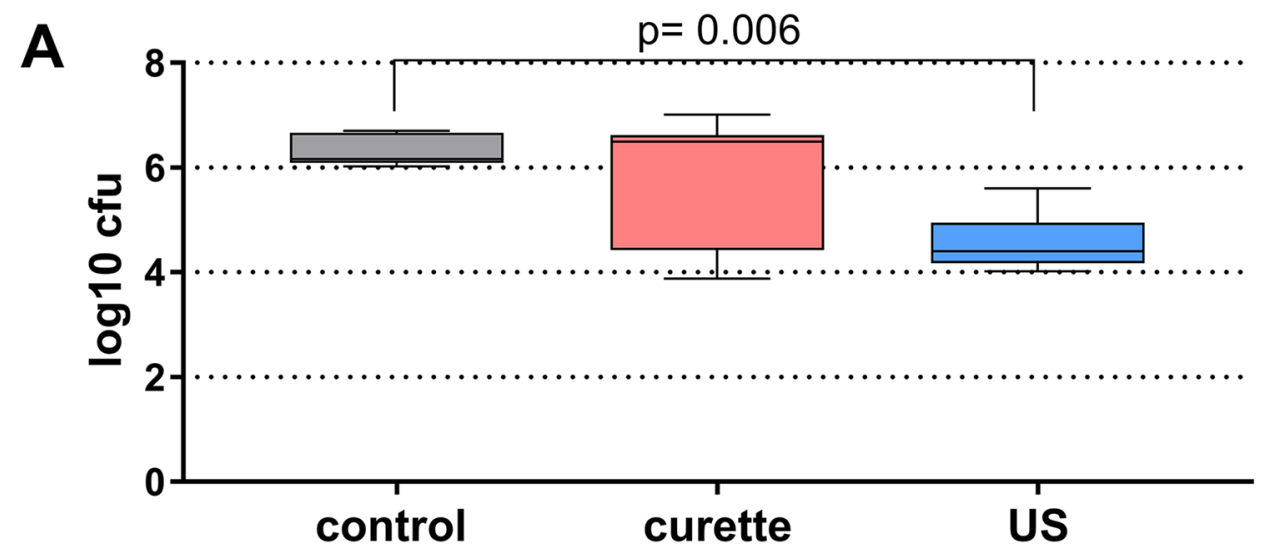

B

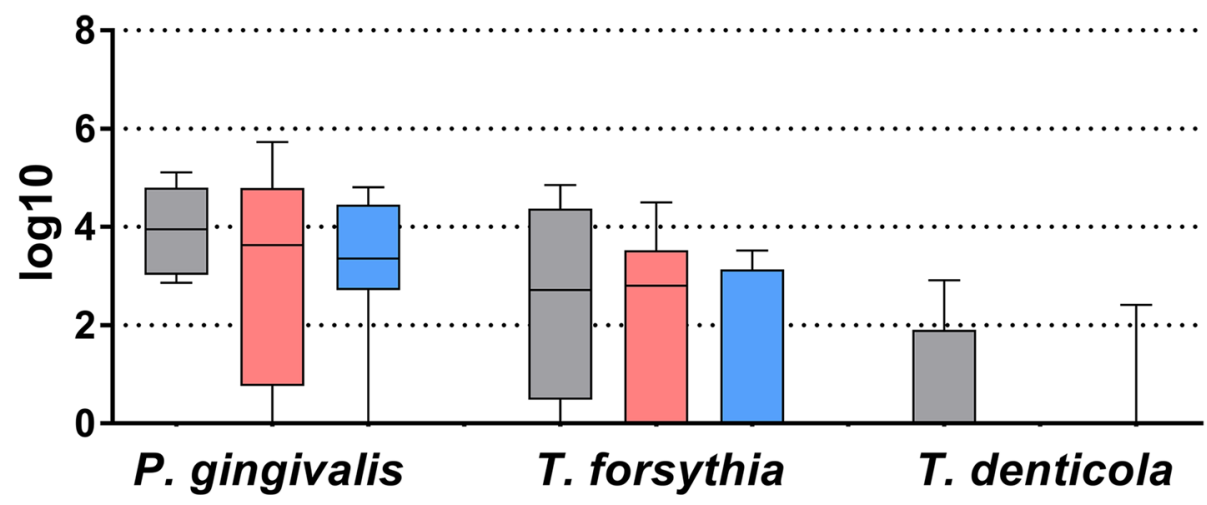

all of the test biofilm samples. T. forsythia was found in six and T. denticola in two of the eight control samples. Lower detection levels were obtained for both test groups although without any statistically significant difference when comparing the three groups. By trend, counts of $T$. forsythia were lower after US (Fig. 2B).

\section{Interaction with epithelial cells}

Four out of eight samples from the control and the curette group were tested positively for colony-forming units attaching or invading epithelial cells. $P$. gingivalis was detected in three controls and in four samples after instrumentation with curettes. Conversely, after ultrasonic instrumentation, only in one sample bacteria could be cultured. $P$. gingivalis was not identified at all, and $T$. denticola, which is not cultivable on regular blood agar plates, was detected in two samples. When comparing the counts of total cfu of selected bacteria, there was however no difference between the groups (Fig. 3A and B).

\section{IL-8 expression}

IL-8 was quantified in the cell supernatants of epithelial cells $6 \mathrm{~h}$ after applying instrumentation to the biofilms. The difference between the three groups was statistically significant $(p=0.010)$. The followed up analysis found significantly lower IL-8 levels when a biofilm treated with US was exposed to epithelial cells in comparison with an untreated control $(p=0.003)$. Although the levels seemed to decrease after biofilm treatment with hand instruments in contact with epithelial cells, this difference failed statistical significance (Fig. 4).

\section{Discussion}

In this study, we sought to develop a pocket model integrating epithelial cells and a multispecies pathogenic biofilm aiming to investigate the cell-biofilm interactions following treatment with two different (i.e., hand curettes or ultrasonic) instrumentation modalities. 
Fig. 3 Median, quartiles, and range of total colony-forming units (cfu; $\mathbf{A})$ and counts of selected bacterial species (determined by real-time PCR; B) adhering or invading epithelial cells $6 \mathrm{~h}$ after applying hand ultrasonic scaler to biofilms on dentin discs instrumentation (curette) and an

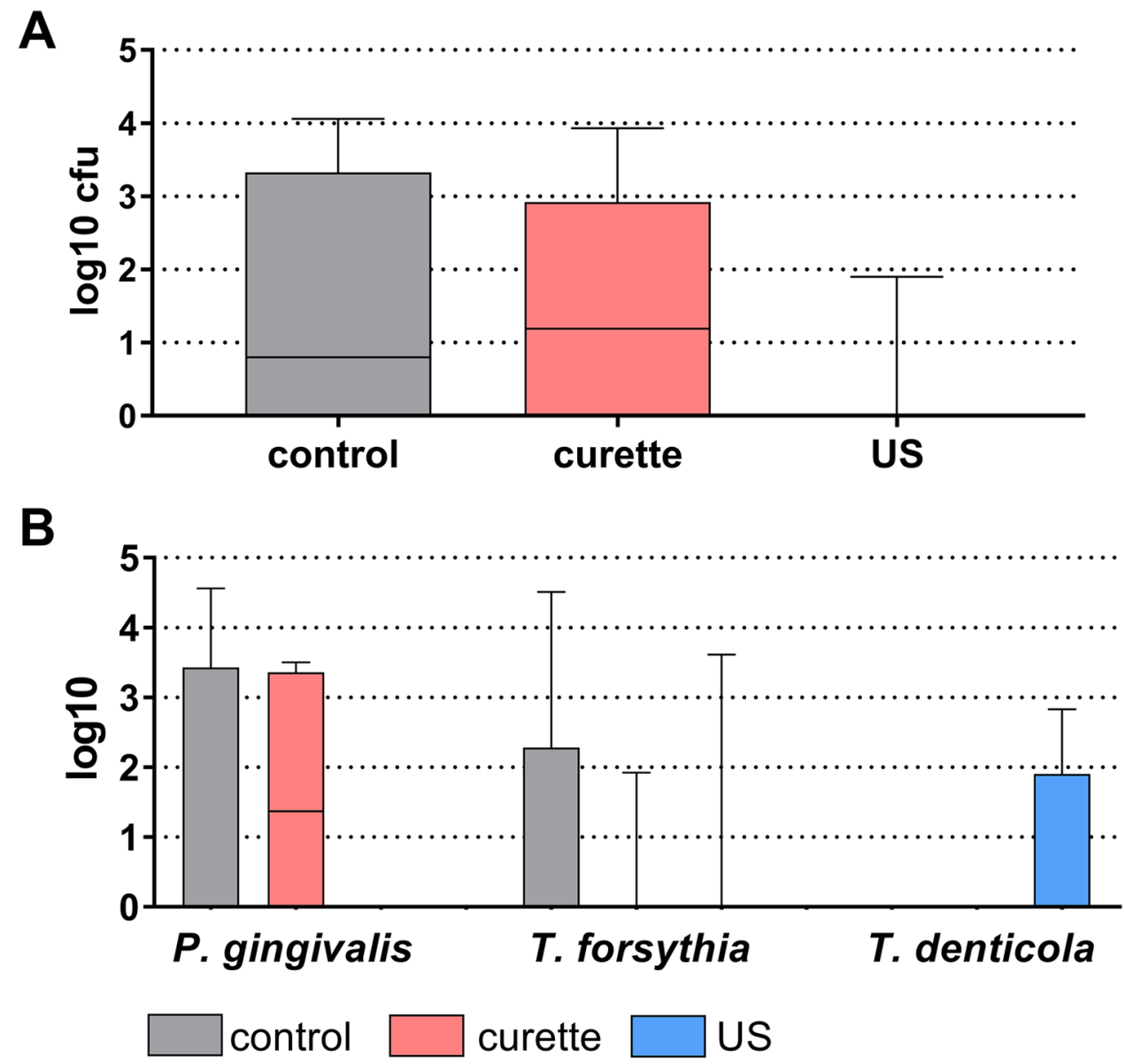

Fig. 4 Interleukin-8 level (median, quartiles, and range) released from epithelial cells $6 \mathrm{~h}$ after applying hand instrumentation (curette) and an ultrasonic scaler to biofilms on dentin discs

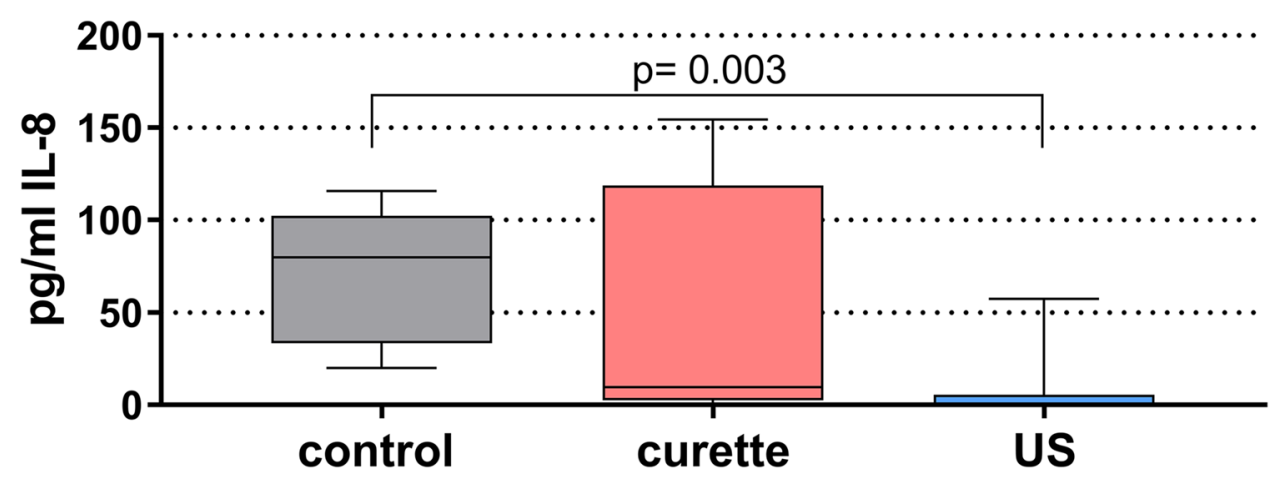

A few in vitro studies analyzed the activity of therapeutics both on periodontal biofilm and on epithelial cells $[22,23,32]$; their purpose was to evaluate the anti-biofilm activity along with the therapeutics' biocompatibility. Recently, a model has been developed to study the interaction of biofilm with epithelial cells when applying antiseptic natural oral health care products [25]. Similar to our model, biofilm was cultured first and independently then brought in close contact (without touching) to epithelial cells, and finally the test products were added to the system. In our model, however, it was impossible to apply instrumentation without touching and damaging the epithelial monolayer. Therefore, we decided to perform the instrumentation in the model first before adding the epithelial cells in order to not mechanically disturb the cells which might be considered as a limitation. All analyses were made $6 \mathrm{~h}$ after instrumentation when changes in the biofilm have already occurred: $T$. denticola was not detected in any of the controls; $P$. gingivalis was highly present in the biofilm composition, very often also after instrumentation. Treponema spp. are known for their extracellular location and close contact to epithelium [38]. Although it is well known that a biofilm consists of microorganisms and the surrounding biofilm matrix, we 
focused only on cfu analysis here. This might be related to the multitude of analyses to be performed at the same time. A more comprehensive biofilm analysis should be included in upcoming research.

The focus of the interaction between the biofilm after instrumentation and epithelial cells was put on the adhesion and invasion of bacteria and on the release of interleukin-8. Thereby, we did not differentiate between adhered and intracellularly located bacteria. Invasion of $P$. gingivalis and $T$. forsythia was studied several times [20,28]; in the case of $P$. gingivalis, the activity of Arg-gingipains as a major virulence factor seems to be of importance [30]. As the method used for determining adhered incl. invasive bacteria destroys the epithelial cells, only released molecules could be used to assess the inflammatory response by the epithelial cells.

Given the inhibitory effect of $P$. gingivalis on IL- 8 levels-it induces the degradation of IL- 8 by gingipain proteases, a phenomenon called "local chemokine paralysis"-[6] a lower detection rate of P.gingivalis attached to or invaded into epithelial cells should result in higher IL-8 expression of the cells. However, this was not the case in our experiments. Epithelial cells exposed to discs after ultrasonic instrumentation exhibited a reduced IL-8 expression together with lower detection rates of $P$.gingivalis compared to control and hand instrumentation.

Considering that this is an in vitro model, it should be kept in mind that in an in vivo scenario the interplay of cells and biofilm depends on many more players on both sides with complex secretion patterns of virulence factors, endotoxins, and host cytokines. Moreover, the inflammatory status of the pocket epithelium along with irregularities on the root surface is likely to influence the treatment itself. Of course, in an in vitro situation, this complexity is lacking, and variances in host immune response or local site anatomy might disguise potential effects of each treatment approach. Also, standardized dentin discs were produced lacking any kind of irregularities. Nevertheless, this model mimicking a periodontal pocket provides an experimental setting to expose different instrumentation modalities, biofilm-inoculated dentin discs with epithelial cells. When thinking further along this path, a 3D model combining epithelial and other cell types (fibroblasts, monocytic cells) might be valuable.

Even though it has been well established that manual and ultrasonic instrumentation result in similar clinical outcomes in periodontitis patients $[1,17,31]$, the two treatment modalities might influence the biofilm-cell interactions on a molecular level. Differences in biofilm removal 14 or water irrigation are likely to induce different cell responses of surrounding epithelial cells. The main findings of this study are that US instrumentation reduced the cfu counts significantly more than HI did together with less IL-8 release from epithelial cells compared to HI.
Our results showed that both treatment modalities substantially reduced bacterial counts, however, US to a greater extent than manual instrumentation. $P$. gingivalis was consistently found in nearly all samples. This is not surprising as $P$. gingivalis was proven to be hard to eliminate even after non-surgical mechanical therapy combined with systemic antibiotics [16, 27]. In our samples, T. forsythia was found in the majority of the control and to lower extent also in the test samples. Our detection rates agree with previously reported data by a clinical study. Within this context, $T$. forsythia was detected between 6.6 and $13.3 \%$ of sites in periodontitis patients after ultrasonic instrumentation [34]. In our study, T. denticola was found in only few samples of the control group and in even fewer of both test group samples. This is lining up with findings from a 2006 randomized clinical trial including patients diagnosed with former generalized aggressive periodontitis of whom subgingival samples were checked for periodonto-pathogenic bacteria using checkerboard DNA - DNA hybridization [36]. SRP alone resulted in a significant reduction of bacteria. T. denticola was identified at baseline in $81.8 \%$ of the patients and 6 weeks after SRP in $36.3 \%$ of the patients. This percentage was maintained up to the end of the follow-up time of 6 months. While these studies did not focus on hand versus ultrasonic instrumentation, Ioannou and co-workers found a significantly greater reduction of T. forsythia and T. denticola after 6 months favoring manual over ultrasonic instrumentation [14].

Next, we looked at the IL-8 expression of the epithelial cells. Here, US resulted in significantly lower IL-8 secretion by epithelial cells compared to the untreated control. IL-8 is a potent chemoattractant for neutrophils and responsible for neutrophil-induced tissue destruction [15]. It has been shown that IL-8 is elevated in periodontitis patients but is also being expressed in healthy controls. Its levels can be reduced by periodontal therapy [10]. In a clinical study, mechanical instrumentation resulted in a significant decrease of IL-8 levels in gingival crevicular fluid [21]. However, the interleukin levels did not correlate with the clinical outcomes. It has to be stressed out that instrumentation was performed using a combination of hand and ultrasonic instruments. In smokers with periodontal disease, nonsurgical mechanical therapy (also here not further specified) induced a significantly higher increase of IL-8 compared to non-smokers with periodontal disease [33]. When comparing hand versus ultrasonic instrumentation in terms of inflammatory markers, no differences between IL-6, CRP, and TNF- $\alpha$ levels at days 1 and 7 after instrumentation were discerned [17]. Also, numerous in vitro studies showed that human gingival fibroblasts secrete increased levels of IL-8 in response to LPS from $P$. gingivalis [3, 13, 24]. As such, a recent study combined a three-dimensional gingival model with biofilms associated with both gingival health and gingivitis. They could show that cytotoxicity and IL-8 expression 
were rising with increasing maturity of the biofilm [4]. And yet, limited evidence exists on models integrating biofilmcell interactions in different treatment modalities as here was done.

The clinical relevance of this in vitro study should be interpreted with caution. The transition from health to disease is accompanied by quantitative and qualitative alteration in the biofilm composition, and therefore, even the alteration in the commensal fraction of the biofilm could play a crucial part. However, a limitation of this study was that we concentrated on a few periodonto-pathogenic bacteria. Yet, our results imply that biofilm-cell interactions could be substantially influenced by different treatment modalities and in particular in view of the different IL- 8 secretion might result in different tissue destruction. In the clinic, however, both manual and ultrasonic instrumentation are often combined making it difficult to see effects emerging from the treatment modality per se. This is also true for a vast number of studies, which often combined manual and ultrasonic instrumentation or missed to specify "SRP." Our findings presented here leave many questions unanswered as for example to what extent water irrigation influenced the result or whether other cytokines relevant for periodontal inflammation or healing might be impacted. Further research should aim to establish well-suited models to further test the effects of different treatment modalities on cell-biofilm interactions and to increase the understanding of these complex interactions by including other cell types within periodontal pockets.

Acknowledgements We thank Prashanthnj Sivapatham for technical assistance.

Funding Open access funding provided by University of Bern. This study was funded by the Department of Periodontology, University of Bern, Switzerland.

\section{Declarations}

Ethics approval The dentin discs used for the experiments were retrieved from de-identified human teeth. The Ethics Committee of the Kanton Bern has confirmed that no ethical approval is required.

Informed consent Written informed consent was obtained from all donors of teeth that were extracted.

Conflict of interest The authors declare no competing interests.

Open Access This article is licensed under a Creative Commons Attribution 4.0 International License, which permits use, sharing, adaptation, distribution and reproduction in any medium or format, as long as you give appropriate credit to the original author(s) and the source, provide a link to the Creative Commons licence, and indicate if changes were made. The images or other third party material in this article are included in the article's Creative Commons licence, unless indicated otherwise in a credit line to the material. If material is not included in the article's Creative Commons licence and your intended use is not permitted by statutory regulation or exceeds the permitted use, you will need to obtain permission directly from the copyright holder. To view a copy of this licence, visit http://creativecommons.org/licenses/by/4.0/.

\section{References}

1. Badersten A, Nilveus R, Egelberg J (1984) Effect of nonsurgical periodontal therapy. II. Severely advanced periodontitis. J Clin Periodontol 11:63-76

2. Bao K, Papadimitropoulos A, Akgul B, Belibasakis GN, Bostanci $\mathrm{N}$ (2015) Establishment of an oral infection model resembling the periodontal pocket in a perfusion bioreactor system. Virulence 6:265-273

3. Bender O, Weinberg E, Moses O, Nemcovsky CE, Weinreb M (2020) Porphyromonasgingivalis lipopolysaccharide and glycated serum albumin increase the production of several pro-inflammatory molecules in human gingival fibroblasts via NFkappaB. Arch Oral Biol 116: 104766

4. Brown JL, Johnston W, Delaney C, Rajendran R, Butcher J, Khan S, Bradshaw D, Ramage G, Culshaw S (2019) Biofilm-stimulated epithelium modulates the inflammatory responses in co-cultured immune cells. Sci Rep 9:15779

5. Buskermolen JK, Janus MM, Roffel S, Krom BP, Gibbs S (2018) Saliva-derived commensal and pathogenic biofilms in a human gingiva model. J Dent Res 97:201-208

6. Darveau RP, Belton CM, Reife RA, Lamont RJ (1998) Local chemokine paralysis, a novel pathogenic mechanism for Porphyromonas gingivalis. Infect Immun 66:1660-1665

7. de Mendonca AC, Maximo MB, Rodrigues JA, Arrais CA, de Freitas PM, Duarte PM (2008) Er:YAG laser, ultrasonic system, and curette produce different profiles on dentine root surfaces: an in vitro study. Photomed Laser Surg 26:91-97

8. Drisko CH (1998) Root instrumentation. Power-driven versus manual scalers, which one? Dent Clin North Am 42:229-244

9. Eick S, Straube A, Guentsch A, Pfister W, Jentsch H (2011) Comparison of real-time polymerase chain reaction and DNA-strip technology in microbiological evaluation of periodontitis treatment. Diagn Microbiol Infect Dis 69:12-20

10. Gamonal J, Acevedo A, Bascones A, Jorge O, Silva A (2001) Characterization of cellular infiltrate, detection of chemokine receptor CCR5 and interleukin-8 and RANTES chemokines in adult periodontitis. J Periodontal Res 36:194-203

11. Gellin RG, Miller MC, Javed T, Engler WO, Mishkin DJ (1986) The effectiveness of the Titan-S sonic scaler versus curettes in the removal of subgingival calculus A human surgical evaluation. $\mathbf{J}$ Periodontol 57:672-680

12. Hagi TT, Klemensberger S, Bereiter R, Nietzsche S, Cosgarea R, Flury S, Lussi A, Sculean A, Eick S (2015) A biofilm pocket model to evaluate different non-surgical periodontal treatment modalities in terms of biofilm removal and reformation, surface alterations and attachment of periodontal ligament fibroblasts. PLoS One 10: e0131056

13. Hayata M, Watanabe N, Kamio N, Tamura M, Nodomi K, Tanaka K, Iddamalgoda A, Tsuda H, Ogata Y, Sato S, Ueda K, Imai K (2019) Cynaropicrin from Cynara scolymus L. suppresses Porphyromonas gingivalis LPS-induced production of inflammatory cytokines in human gingival fibroblasts and RANKLinduced osteoclast differentiation in RAW264.7 cells. J Nat Med 73:114-123

14. Ioannou I, Dimitriadis N, Papadimitriou K, Sakellari D, Vouros I, Konstantinidis A (2009) Hand instrumentation versus ultrasonic debridement in the treatment of chronic periodontitis: a 
randomized clinical and microbiological trial. J Clin Periodontol $36: 132-141$

15. Jin LJ, Leung WK, Corbet EF, Soder B (2002) Relationship of changes in interleukin-8 levels and granulocyte elastase activity in gingival crevicular fluid to subgingival periodontopathogens following non-surgical periodontal therapy in subjects with chronic periodontitis. J Clin Periodontol 29:604-614

16. Johnson JD, Chen R, Lenton PA, Zhang G, Hinrichs JE, Rudney JD (2008) Persistence of extracrevicular bacterial reservoirs after treatment of aggressive periodontitis. J Periodontol 79:2305-2312

17. Johnston W, Paterson M, Piela K, Davison E, Simpson A, Goulding M, Ramage G, Sherriff A, Culshaw S (2020) The systemic inflammatory response following hand instrumentation versus ultrasonic instrumentation-a randomized controlled trial. J Clin Periodontol 47:1087-1097

18. Kantrong N, To TT, Darveau RP (2019) Gingival epithelial cell recognition of lipopolysaccharide. Adv Exp Med Biol 1197:55-67

19. Khosravi M, Bahrami ZS, Atabaki MS, Shokrgozar MA, Shokri F (2004) Comparative effectiveness of hand and ultrasonic instrumentations in root surface planing in vitro. J Clin Periodontol 31:160-165

20. Kirschbaum M, Schultze-Mosgau S, Pfister W, Eick S (2010) Mixture of periodontopathogenic bacteria influences interaction with KB cells. Anaerobe 16:461-468

21. Konopka L, Pietrzak A, Brzezinska-Blaszczyk E (2012) Effect of scaling and root planing on interleukin-1beta, interleukin- 8 and MMP-8 levels in gingival crevicular fluid from chronic periodontitis patients. J Periodontal Res 47:681-688

22. LeBel G, Vaillancourt K, Morin MP, Grenier D (2020) Antimicrobial activity, biocompatibility and anti-inflammatory properties of cetylpyridinium chloride-based mouthwash containing sodium fluoride and xylitol: an in vitro study. Oral Health Prev Dent 18:1069-1076

23. Lim SY, Dafydd M, Ong J, Ord-McDermott LA, Board-Davies E, Sands K, Williams D, Sloan AJ, Heard CM (2020) Mucoadhesive thin films for the simultaneous delivery of microbicide and antiinflammatory drugs in the treatment of periodontal diseases. Int $\mathbf{J}$ Pharm 573: 118860

24. Liu W, Huang G, Yang Y, Gao R, Zhang S, Kou B (2021) Oridonin inhibits epithelial-mesenchymal transition of human nasopharyngeal carcinoma cells by negatively regulating AKT/STAT3 signaling pathway. Int J Med Sci 18:81-87

25. Millhouse E, Jose A, Sherry L, Lappin DF, Patel N, Middleton AM, Pratten J, Culshaw S, Ramage G (2014) Development of an in vitro periodontal biofilm model for assessing antimicrobial and host modulatory effects of bioactive molecules. BMC Oral Health 14:80

26. Mlachkova A, Popova C, Doseva V (2020) Presence of IL-8 gene polymorphism and IL-8 serum levels in patients with chronic periodontitis - literature review. Folia Med (Plovdiv) 62:253-257
27. Mombelli A, Schmid B, Rutar A, Lang NP (2000) Persistence patterns of Porphyromonas gingivalis, Prevotella intermedia/nigrescens, and Actinobacillus actinomyetemcomitans after mechanical therapy of periodontal disease. J Periodontol 71:14-21

28. Rudney JD, Chen R, Sedgewick GJ (2005) Actinobacillus actinomycetemcomitans, Porphyromonas gingivalis, and Tannerella forsythensis are components of a polymicrobial intracellular flora within human buccal cells. J Dent Res 84:59-63

29. Schmidlin PR, Beuchat M, Busslinger A, Lehmann B, Lutz F (2001) Tooth substance loss resulting from mechanical, sonic and ultrasonic root instrumentation assessed by liquid scintillation. $\mathbf{J}$ Clin Periodontol 28:1058-1066

30. Takeuchi H, Amano A (2021) Invasion of gingival epithelial cells by Porphyromonas gingivalis. Methods Mol Biol 2210:215-224

31. Thompson JA, Lee DJ, Lindgren CG, Benz LA, Collins C, Levitt D, Fefer A (1988) Influence of dose and duration of infusion of interleukin-2 on toxicity and immunomodulation. J Clin Oncol 6:669-678

32. Varghese J, Ramenzoni LL, Shenoy P, Nayak UY, Nayak N, Attin $T$, Schmidlin PR (2019) In vitro evaluation of substantivity, staining potential, and biofilm reduction of guava leaf extract mouth rinse in combination with its anti-inflammatory effect on human gingival epithelial keratinocytes. Materials (Basel) 12

33. Vijayakumar S, Koshi E, Sadasivan A, Indhuja RS, Vallabhan CG (2020) Effect of nonsurgical periodontal therapy on serum levels of interleukin-1beta and interleukin- 8 in smokers and nonsmokers with chronic periodontitis. J Pharm Bioallied Sci 12:S313-S318

34. Wadhwani RB, Chaudhary MS, Tharani DA, Chandak SA (2013) Effect of scaling and root planing on detection of $<\mathrm{i}>$ Tannerella forsythia $<$ i $>$ in chronic periodontitis. J Oral Dis 2013: 383746

35. Walmsley AD, Lea SC, Landini G, Moses AJ (2008) Advances in power driven pocket/root instrumentation. J Clin Periodontol $35: 22-28$

36. Xajigeorgiou C, Sakellari D, Slini T, Baka A, Konstantinidis A (2006) Clinical and microbiological effects of different antimicrobials on generalized aggressive periodontitis. J Clin Periodontol 33:254-264

37. Zappa U, Smith B, Simona C, Graf H, Case D, Kim W (1991) Root substance removal by scaling and root planing. J Periodontol 62:750-754

38. Zijnge V, van Leeuwen MB, Degener JE, Abbas F, Thurnheer T, Gmur R, Harmsen HJ (2010) Oral biofilm architecture on natural teeth. PLoS One 5: e9321

Publisher's Note Springer Nature remains neutral with regard to jurisdictional claims in published maps and institutional affiliations. 